\title{
Otitis media con efusión en pacientes con fisura palatina: Comparación de estrategias terapéuticas
}

\author{
Otitis media with effusion in patients with cleft palate: \\ Comparison of treatment strategies
}

\author{
Michel Royer $\mathrm{F}^{1,2}$, Ornella Dorador $\mathrm{G}^{3}$, Mirta Palomares $\mathrm{A}^{4,5}$, Ursula Zelada $\mathrm{B}^{6}$, \\ Drina Álvarez $\mathbf{C}^{5}$, Carolina Villena $\mathbf{B}^{5}$.
}

\begin{abstract}
RESUMEN
Introducción: La otitis media con efusión presenta una alta prevalencia en pacientes con fisura palatina, debido a una obstrucción funcional de la tuba auditiva. Dada su asociación con hipoacusia y una baja tendencia de resolución espontánea, existe controversia sobre la necesidad de instalar tubos de ventilación en estos pacientes.

objetivo: Comparar resultados anatómicos y audiológicos de dos estrategias de tratamiento.

Material y método: Estudio de cohorte histórica en niños de 8 a 11 años con fisura velopalatina operada. La otitis con efusión de un grupo se trató con tubos de ventilación, mientras otro grupo tuvo un manejo conservador. La presencia de alteraciones timpánicas e hipoacusia se compararon entre ambos grupos mediante Xi cuadrado y test de Fischer.

Resultados: Cincuenta y seis pacientes fueron reclutados, 24 en estrategia quirúrgica y 32 en conservadora. Todos los pacientes presentan una evaluación fonoaudiológica normal. Existe mayor frecuencia de retracción timpánica, perforación timpánica e hipoacusia de conducción en aquellos niños sometidos a una estrategia agresiva de tratamiento.

Discusión: Existen mayores alteraciones timpánicas e hipoacusia en los pacientes sometidos a una estrategia de tratamiento quirúrgica, por lo que parece prudente realizar una evaluación individual y determinar la necesidad de cirugía de acuerdo a cada paciente.
\end{abstract}

Palabras clave: Otitis media, Fisura labiopalatina, Tubos de ventilación.

\section{ABSTRACT}

Introduction: Otitis media with effusion (OME) is a frequent finding in patients with cleft palate due to a functional obstruction of the Eustachian tube. Because of its

\footnotetext{
Médico. Servicio Otorrinolaringología. Hospital Dr. Luis Calvo Mackenna.

Médico. Unidad Otorrinolaringología, Fundación Gantz - Hospital del Niño con Fisura.

Fonoaudióloga. Hospital Regional de Antofagasta Dr. Leonardo Guzmán.

Fonoaudióloga. Hospital Dr. Luis Calvo Mackenna.

Fonoaudióloga. Fundación Gantz - Hospital del Niño con Fisura.

Médico. Servicio Otorrinolaringología, Hospital Barros Luco Trudeau.
} 
association with hearing loss and low rate of spontaneous resolution the use of ventilation tubes as treatment appears as beneficial. The clinical and audiological consequences of this conduct are not well established.

Aim: To compare anatomical and audiologics results with two strategies of treatment.

Material and method: Historical cohort study of children from 8 to 10 years, with cleft palate, surgically corrected, and with OME. One group was treated with ventilation tubes, and an expectant management was carried out with the other group. The following parameters were compared by Xi-square test and Fischer: tympanic membrane state, presence of complications and hearing level.

Results: Fifty six patients were admitted, 24 in surgical management and 32 in conservative. Every child had a cleft palate corrected without velopharyngeal insufficiency. It was found a higher frequency of tympanic membrane retraction, tympanic membrane perforation and hearing loss in the case groups were found.

Discusion: We found a greater presence of tympanic alterations and hearing disorders in patients subjected to ventilation tubes as treatment of OME. It seems prudent to evaluate the surgical alternative individually according to each patient characteristic.

Key words: Otitis media, Cleft palate, Grommet.

\section{INTRODUCCIÓN}

La otitis media con efusión (OME), definida como presencia de líquido en el oído medio sin signos de infección, es una patología de alta prevalencia en pacientes portadores de fisura palatina, con cifras estimadas sobre el $90 \%$, incluso tras la reparación quirúrgica de la fisura ${ }^{1,2}$. Esta condición se produce por una obstrucción funcional de la tuba auditiva, debido a alteraciones tanto en la inserción como en el funcionamiento del músculo tensor del velo del paladar, elemento clave en la apertura de la trompa. Tras el cierre quirúrgico de la fisura no se logra una función muscular completamente normal, por lo que la disfunción tubaria y por consecuencia la OME tiende a persistir en el tiempo, con una baja tasa de resolución espontánea ${ }^{3-6}$.

La OME conlleva una hipoacusia de conducción, en general de grado leve a moderado, y debido a que esta disminución de la percepción auditiva se presenta en un período crítico de la adquisición de las habilidades de lenguaje y habla, existe una razonable preocupación en cuanto al desarrollo de estos niños?

En esta disyuntiva, han surgido dos corrientes de tratamiento de la OME. Una quirúrgica, que busca evitar la hipoacusia de conducción y las eventuales complicaciones otológicas de su persistencia, realizando una inserción temprana de tubos de ventilación, en el momento de la palatoplastia, y reintervenciones ante la aparición de efusión durante el seguimiento ${ }^{8}$. Una segunda estrategia, más conservadora, basada en que la mayoría de las OME son asintomáticas, busca evitar las complicaciones de las inserciones repetidas de tubos de ventilación y disminuir los costos, mediante la selección de pacientes a quienes realizar la intervención quirúrgica ${ }^{9}$.

En experiencias internacionales, los partidarios de una conducta de tratamiento quirúrgica han demostrado que niños con fisura palatina y OME tratada con tubos de ventilación, comparados a niños sin fisura, no presentan diferencias en la prevalencia de OME, y las características del tímpano y audiológicas son similares ${ }^{10,11}$.

Otros estudios, con una estrategia conservadora de tratamiento, han demostrado que sólo $28 \%$ de los niños con fisura palatina requiere inserción de tubos de ventilación por hipoacusia significativa, otitis media a repetición 0 retracción timpánica ${ }^{12,13}$. También se ha demostrado una mayor frecuencia de otitis media aguda, otitis media con efusión y perforaciones timpánicas en el grupo con tratamiento quirúrgico ${ }^{14,15}$. Además, la mayor frecuencia de alteraciones clínicas o complicaciones se concentran en el grupo de pacientes que ha requerido 3 o más inserciones de tubos de ventilación ${ }^{15,16}$.

En Chile, la fisura palatina es una patología incluida en el plan de garantías explícitas en salud 
(GES), por lo que tiene un abordaje multidisciplinario, con una guía clínica, en un cronograma establecido. En lo referente a OME, la guía chilena plantea un tratamiento con amoxicilina, conducta expectante al año e indicación de tubos de ventilación en niños sobre 2 años con OME persistente. En el seguimiento, tras la caída del tubo de ventilación, al tercer mes se controla el estado del oído medio y de persistir efusión se instala un tubo ventilación de larga duración (en T). Además, se considera que la presencia de retracción severa 0 asociada a hipoacusia debiera ser tratada de esta misma forma ${ }^{17}$. Dada la experiencia internacional y la sugerencia indicada por la guía clínica chilena, pueden encontrarse diferentes estrategias de tratamiento en nuestro país.

\section{OBJETIVO}

El objetivo de este trabajo es comparar los resultados anatómicos y funcionales de una conducta quirúrgica 0 conservadora en el manejo de otitis media con efusión en niños con fisura palatina.

\section{MATERIAL Y MÉTODO}

La fisura del paladar en Chile es una patología incluida en el programa GES, por lo que este estudio consideró dos centros de Santiago capacitados en este tipo de patología.

Ambas instituciones diferían en su conducta habitual de tratamiento de OME en niños portadores de fisura palatina. En el Centro 1 se realizaba instalación temprana de tubos de ventilación (momento de palatoplastía) y reintervención ante la existencia de efusión durante el seguimiento, lo que denominamos conducta quirúrgica. En el Centro 2 se realizaba seguimiento clínico a los niños y la indicación de tubos de ventilación se restringía a aquellos que presentaban otitis media a repetición (más de 3 episodios en 6 meses), otitis fibroadhesiva (retracción timpánica) y/o hipoacusia clínicamente significativa (umbral de vía aérea sobre $30 \mathrm{~dB}$ en audiometría y dificultades en vida diaria referidas por paciente o familiar), lo que denominamos una conducta conservadora.

Se realiza un estudio de cohorte histórica, incluyendo niños entre 8 a 11 años, con antece- dente de fisura labiopalatina unilateral tratada quirúrgicamente en forma adecuada. Se excluyen aquellos pacientes con fisura asociada a algún síndrome genético y quienes presentaran insuficiencia velofaríngea tras la reparación de la fisura. Se escoge el rango etario descrito, dado que se quiere evaluar el estado anatómico y funcional del oído tras el período en que las patologías agudas tienen mayor incidencia. Para el reclutamiento, realizado entre junio a septiembre de 2008, se contactó telefónicamente a los cuidadores, con el fin de realizar un control no programado previamente ni impulsado por sintomatología concurrente.

A cada paciente en forma individual se le realiza una evaluación otorrinolaringológica, fonoaudiológica y una audiometría tonal. La evaluación otorrinolaringológica consta de una anamnesis, revisión de historia clínica y otomicroscopía. Los hallazgos de la otomicroscopía se clasificaron en: normal, miringoesclerosis, retracción según clasificación de Sadé18, perforación y colesteatoma. La evaluación de fonoaudiología de habla y lenguaje consideró aspectos de articulación y resonancia. La audiometría tonal se realizó de forma estándar y se consideraron para los análisis los valores promedios de umbrales vía ósea y aérea de las frecuencias $500,1.000$ y $2.000 \mathrm{~Hz}$.

Los resultados se analizan estadísticamente con la prueba de Xi cuadrado o test de Fischer, considerándose significativo un valor de $\mathrm{p}$ menor a 0,05.

\section{RESULTADOS}

Durante el período establecido se logró reclutar a 56 pacientes, perteneciendo 24 al Centro 1 (conducta quirúrgica) y 32 al Centro 2 (conducta conservadora). Todos tenían el antecedente de una fisura palatina corregida quirúrgicamente sin insuficiencia velofaríngea posterior. La evaluación fonoaudiológica mediante pruebas de resonancia y articulación de lenguaje resultó normal en todos los niños, sin necesidad actual de tratamiento foniátrico.

Al evaluar el número de intervenciones por paciente, existió una mayor frecuencia de instalación de tubos de ventilación en el Centro 1 (Figura 1), destacando que el $70 \%$ de sus pacientes fueron sometidos a 2 o más intervenciones, mientras el $59 \%$ de los niños del Centro 2 no fue sometido a cirugía. 
En cuanto a los hallazgos al examen timpánico (Figura 2) destaca 40\% de retracción en los oídos de los pacientes del Centro 1 y $16 \%$ en el Centro 2 en este mismo ítem. Esta diferencia es estadísticamente significativa $p \leq 0,01$, así también la establecida en presencia de perforación timpánica, $23 \%$ en la estrategia quirúrgica y $2 \%$ en la conservadora.

En la evaluación audiológica, 18 oídos del Centro 1 presentan hipoacusia de conducción y 8 del Centro 2, siendo esta diferencia estadísticamente significativa $p \leq 0,01$ (Figura 3 ). La mayoría de las hipoacusias diagnosticadas corresponden a un grado leve, siendo en grado moderado 4 oídos del Centro 1 y 2 oídos del Centro 2.

\section{DISCUSIÓN}

La otitis media con efusión es una patología que provoca hipoacusia de conducción a temprana edad, por lo que se ha discutido su posible impacto en el desarrollo del lenguaje en los niños. Estudios previos en niños sin otra patología, han demostrado que la presencia de OME no influye en las capacidades globales de lenguaje, por lo que se aconseja una postura conservadora frente a esta patología $a^{19,20}$.

En la mayoría de los estudios y consensos de OME se excluyen los pacientes con fisura palatina, por la comorbilidad y la tendencia a ser persistente en el tiempo, por lo que existe una mayor preocupación por su posible impacto en la adquisición de habilidades de habla $y$ lenguaje en estos pacientes.

Desde el extranjero han surgido dos estrategias de tratamiento, una activamente quirúrgica, que ante la presencia de OME indica instalación de tubos de ventilación ${ }^{8}$, y otra más conservadora en la cual se seleccionan los pacientes candidatos a cirugía ${ }^{9}$. En Chile, existe una guía clínica, pero en la práctica existen diferentes realidades en cuanto a elección de tratamiento. Por ello, ante la posibilidad de comparar dos centros con experiencia en la patología, parece interesante demostrar los resultados anatómicos y funcionales a mediano plazo de las estrategias adoptadas.

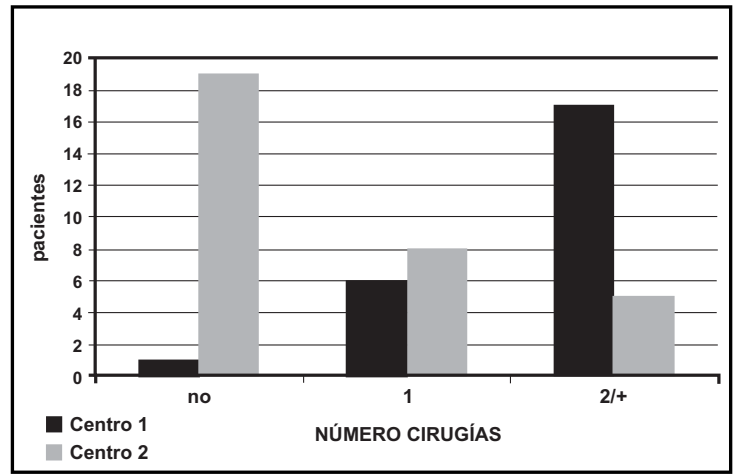

Figura 1. Número de eventos quirúrgicos (instalación tubos de ventilación) por paciente según centro.

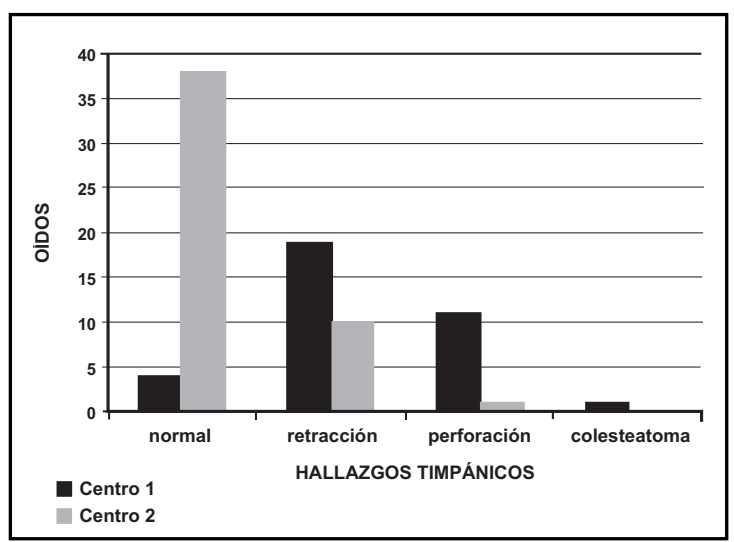

Figura 2. Hallazgos al examen timpánico según estrategia de tratamiento.

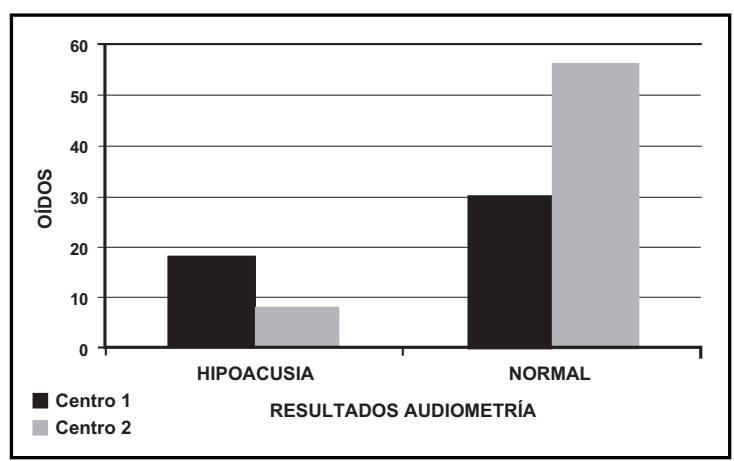

Figura 3. Resultados de evaluación audiológica según estrategia de tratamiento. 
En este protocolo se demuestra que existe una mayor frecuencia de alteraciones anatómicas (retracción y perforación timpánica) en el centro hospitalario con una conducta tradicionalmente más quirúrgica. En este mismo sentido, existe una mayor frecuencia de hipoacusia de conducción en los niños sometidos a esta estrategia de tratamiento. Esto es relevante, no sólo por morbilidad asociada a cada intervención o por aumentar los costos, sino también porque genera pacientes con otro tipo de patología, más compleja y que requieren cirugías adicionales.

Experiencias previas internacionales tienen resultados similares en cuanto a que los pacientes sometidos a mayor número de intervenciones con el fin de mejorar la ventilación del oído medio y la audición, a mediano plazo tienen mayores secuelas anatómicas y funcionales $^{15,16}$. En Chile, se ha descrito un mayor número de alteraciones timpánicas y auditivas en los niños con dos o más inserciones de tubos de ventilación, y en quienes la edad de su primera cirugía de oído fue previo a los cuatro años ${ }^{21}$. En este contexto, no se puede determinar si la enfermedad en estos niños era más agresiva o la inserción repetida de tubos de ventilación provoca las alteraciones, pero sí la cirugía parece ser efectiva en detener la enfermedad o las secuelas de ésta.

Evidentemente existen limitaciones en este tipo de estudio, dado que no pueden fijarse parámetros para el momento de la intervención quirúrgica, sino que se basa en las conductas tradicionales de ambos centros. Esto también ocurre en experiencias internacionales y una reciente revisión de la literatura publicada demostró resultados contradictorios, sin suficiente evidencia para realizar una recomendación adecuada ${ }^{22}$. En este sentido, no puede realizarse una recomendación general para un cambio en la práctica sólo con este nivel de evidencia. De todas formas, las limitaciones en el diseño del estudio tiene el mismo impacto en ambos grupos de pacientes, por lo tanto, según los resultados presentados, el adoptar de forma generalizada una conducta quirúrgica tiene un resultado anatómico y funcional desfavorable frente a una conducta conservadora.

\section{CONCLUSIONES}

De acuerdo a los resultados expuestos, parece más razonable realizar indicaciones individuales sobre la necesidad de cirugía y que no todos los niños con fisura del paladar y efusión en el oído medio deban ser sometidos a inserción de tubos de ventilación. Concomitantemente, sería apropiado avanzar en protocolos prospectivos que demuestren resultados favorables de cada tratamiento para introducir cambios significativos en la conducta clínica frente a estos pacientes.

\section{BIBLIOGRAFIA}

1. Grant HR, Quiney RE, Mercer DM, Lodge S. Cleft palate and glue ear. Arch Dis Child 1988; 63: 176-9.

2. Paradise Jl, Bluestone CD, Felder $H$. The universality of otitis media in 50 infants with cleft palate. Pediatrics 1969; 53: 48-54.

3. Rood SR, DoyLE WJ. The nasopharyngeal orifice of the auditory tube: implications for tubal dynamics anatomy. Cleft Palate J 1982; 19: 119-28.

4. Рвостов B. Anatomy of the Eustachian tube. Arch Otolaryngol 1973; 97: 2-8.

5. Bluestone CD. Eustachian tube obstruction in the infant with cleft palate. Ann Otol Rhinol Laryngol 1971; 80 (Suppl. 2): 1-30.

6. Robinson PJ, Lodge S, Jones BM, et AL. The effect of palate repair on otitis media with effusion. Plast Reconstr Surg 1992; 89: 640-5.

7. Gordon AS, F. Jean-Louis, Morton RP. Late ear sequelae in cleft palate patients. Int $J$ Paediatr Otolaryngol 1988; 15: 149-56.

8. Paradise JL, Bluestone CD. Early treatment of the universal otitis media of infants with cleft palate. Pediatrics 1974; 53: 48-54.

9. Crysdale WS. Rational management of middle ear effusion in the cleft palate patient. J Otol 1976; 5: 463-7.

10. Valtonen H, Dietz A, Qvarnberg Y. Long-term clinical, audiologic, and radiologic outcomes in palate cleft children treated with early tympanostomy for Otitis Media with Effusion: a controlled prospective study. Laryngoscope 2005; 115: 1512-6.

11. Merrick GD, Kunjur J, Watts R, Markus aF. The effect of early insertion of grommets on the development of speech in children with cleft palates. Br J Oral Maxillofac Surg 2007; 45: 527-33. 
12. Shaw R, Richardson D, McMahoin S. Conservative Management of otitis media in cleft palate. J Cranio-Maxillofacial Surgery 2003; 31: 316-20.

13. Robson AK, Blanshard JD, Jones K, Albery EH, SмiтH IM, Maw AR. A conservative approach to the management of otitis media with effusion in cleft palate children. J Laryngol Otol 1992; 106: 788-92.

14. Sheahan P, Blayney AW, Sheahan JN, Earley MJ. Sequelae of otitis media with effusion among children with cleft lip and/or cleft palate. Clin Otolaryngol 2002; 27: 494-500.

15. Phua YS, Salkeld LJ, de Chalain TM. Middle ear disease in children with cleft palate: Protocols for Management. Int J Pediatr Otorhinolaryngol 2009; 73: 307-13.

16. Sheahan P, Miller I, Sheahan JN, Earley MJ, BLAYNEY AW. Incidence and outcome of middle ear disease in cleft lip and/or cleft palate. Int $J$ Pediatr Otorhinolaryngol 2003; 67 (7): 785-93.

17. Ministerio de Salud. Guía Clínica Fisura Labiopalatina. $1^{\text {st }}$ Ed. Santiago: Minsal, 2005.
18. SAde J. The atelactatic ear. En J. Sade (Ed.), Secretory Otitis Media and Its Sequelae, Churchill-Livingstone, New York, 1979; 64-88.

19. Paradise JL, Dollaghan CA, Campbell TF et al. Otitis media and tympanostomy tube insertion Turing the first three years of life: developmental outcomes at the age of four years. Pediatrics 2003; 112(2): 265-77.

20. Paradise Jl, Dollaghan CA, Campbell tF et al. Language, speech sound production, and cognition in three-year-old children in relation to otitis media in their first three years of life. Pediatrics 2000; 105 (5): 1119-30.

21. Dorador 0. Estado auditivo en niños, entre 8 a 11 años de edad, con fisura velo-palatina de dos centros especializados de la región metropolitana. Antofagasta, Chile: Universidad del Mar, 2008. 106 pp.

22. Ponduri S, Bradley R, Ellis PE, Brookes ST, Sandy JR, Ness AR. The management of otitis media with early Soutine insertion of grommets in children with cleft palate - a systematic review. Cleft Palate Craniofac 2009; 46(1): 30-8. 